\title{
Criminologie
}

\section{La responsabilité du génocidaire : entre responsabilité individuelle et responsabilité collective}

\section{Karine Wurtz}

Volume 39, numéro 2, automne 2006

Le crime de génocide : Construction d'un paradigme pour la criminologie, la philosophie et le droit pénal

URI : https://id.erudit.org/iderudit/014428ar

DOI : https://doi.org/10.7202/014428ar

Aller au sommaire du numéro

Éditeur(s)

Les Presses de l'Université de Montréal

ISSN

0316-0041 (imprimé)

1492-1367 (numérique)

Découvrir la revue

Citer cet article

Wurtz, K. (2006). La responsabilité du génocidaire : entre responsabilité individuelle et responsabilité collective. Criminologie, 39(2), 59-76. https://doi.org/10.7202/014428ar
Résumé de l'article

Cet article va interroger la possibilité de concilier à la fois le principe d'individualité de la peine et le caractère collectif du crime de génocide. En effet, face à un crime collectif, nous avons deux risques : ou reprocher à l'individu plus qu'il n'a fait et alors mettre à mal le principe d'individualité de la peine, ou en rester à la stricte description des actes individuels, mais alors la dimension collective du crime est perdue. La réponse nous sera donnée par l'étude de la structure collective originale du crime de génocide. Elle révèle en effet entre les génocidaires un lien tel que chaque acte particulier reflète les autres actes de génocide et n'acquiert sa véritable dimension qu'en référence à ceux-ci. Nous établirons ainsi que la dimension collective du crime et le principe d'individualité de la peine ne sont pas incommensurables.

Il ne s'agit pas dans ce texte de donner un programme de la manière dont il faut traiter un génocidaire lambda lors d'un procès, mais de révéler les structures d'un modèle, pouvant ensuite servir de cadre de référence. 


\title{
La responsabilité du génocidaire : entre responsabilité individuelle et responsabilité collective
}

\author{
Karine Wurtz \\ Doctorante en philosophie \\ Université Montréal et Université de Paris I Panthéon-Sorbone \\ karine.wurtz@umontreal.ca
}

RÉSUMÉ - Cet article va interroger la possibilité de concilier à la fois le principe d'individualité de la peine et le caractère collectif du crime de génocide. En effet, face à un crime collectif, nous avons deux risques: ou reprocher à l'individu plus qu'il n'a fait et alors mettre à mal le principe d'individualité de la peine, ou en rester à la stricte description des actes individuels, mais alors la dimension collective du crime est perdue. La réponse nous sera donnée par l'étude de la structure collective originale du crime de génocide. Elle révèle en effet entre les génocidaires un lien tel que chaque acte particulier reflète les autres actes de génocide et n'acquiert sa véritable dimension qu'en référence à ceux-ci. Nous établirons ainsi que la dimension collective du crime et le principe d'individualité de la peine ne sont pas incommensurables.

Il ne s'agit pas dans ce texte de donner un programme de la manière dont il faut traiter un génocidaire lambda lors d'un procès, mais de révéler les structures d'un modèle, pouvant ensuite servir de cadre de référence.

ABSTRACT - This article is going to question the possibility of reconciling at the same time the principle of individuality of punishment and the collective feature of the crime of genocide. Indeed, in front of a collective crime, we have two risks: or to blame the individual for more than he did and then worsen the principle of individuality of punishment, or to stay at the strict level of the individual acts, but then the collective dimension of the crime is lost. The answer will be brought to us by the study of the original collective structure of the crime of genocide. It indeed reveals between genocidaries a link such as every particular act reflects the other acts of genocide. So, the crime of genocide acquires its real dimension only in reference to these other acts. We shall so establish that the collective dimension of the crime and the principle of individuality of punishment are not incommensurable. It is not a question in this text to give a program of the way we have to treat any genocidary during a trial, but to reveal the structures of a model, which could then serve as reference frame. 
La comparution d'un individu accusé de génocide peut-elle à la fois rendre compte du caractère collectif du crime de génocide tout en respectant le principe d'individualité des peines ou bien devons-nous nécessairement perdre une des deux dimensions au profit de l'autre?

Ce qui nous amène à poser cette question est le constat d'une tension. D'une part, le principe d'individualité de la peine implique que chacun doit payer pour ce qu'il a fait en propre et pour rien de plus que cela. Or, si on ne s'intéresse qu'aux actes propres de l'individu, on ne peut les décrire qu'en termes (par exemple) de meurtre ou, au pis aller, de meurtre aggravé, comme il l'aurait été dans un contexte de crime civil. Ce qui fait la spécificité et l'horreur d'un acte de génocide est alors inévitablement mis de côté.

Mais à l'inverse, si on veut prendre en compte le caractère collectif du crime de génocide pour éclairer et expliquer ce qu'a fait un individu particulier, nous risquons d'avoir du mal à faire des distinctions nettes dans ce qui revient à chacun. Le risque est alors réel d'excuser l'accusé plus que de raison par le renvoi à l'influence contextuelle ou, au contraire, de lui faire porter le poids d'actes qui excèdent les siens propres, ce qui reviendrait à malmener le principe d'individualité de la peine.

Dès lors, l'enjeu de la conciliation de ces deux dimensions est une certaine forme de justice: un individu ne pourra être rétribué équitablement pour ses actes que si on peut prendre en compte la dimension collective de la responsabilité impliquée dans le crime de génocide tout en restant dans les limites de cette dernière.

Mon ambition dans ce texte sera de montrer que cette conciliation est possible, et que la prise en compte de la dimension collective du crime de génocide intervient comme un élément aggravant et non atténuant dans la responsabilité individuelle. Ce qui me permet d'arriver à cette conclusion, et que je me propose de soutenir ici, est l'établissement que chaque acte de génocide est lié de façon structurelle à tous les autres.

Je procéderai donc en quatre étapes: la première visera à démontrer que le crime de génocide est bien un crime collectif; la seconde examinera quel type de collectif peut se livrer au génocide, ce qui nous permettra en troisième partie de dégager les liaisons structurelles qui existent entre les différents génocidaires et d'établir la nature de la responsabilité collective. Enfin, la quatrième et dernière partie estimera les conséquences de ce qui aura été posé quant à la nature de la responsabilité du génocidaire et nous permettra de répondre à la question de 
savoir si le procès d'un individu pour crime de génocide peut ou non concilier responsabilité individuelle et responsabilité collective.

\section{Le crime de génocide est un crime de nature collective}

A priori, le crime de génocide pourrait être vu comme rentrant éminemment dans ce que Peter French (1998: 37) envisageait comme des prédicats ne pouvant s'appliquer qu'à des agents collectifs. Par exemple, le prédicat «perdre une partie de football» ne peut se dire que d'une équipe sportive, le prédicat «élire un président» ne peut se dire que des citoyens d'une nation. Ces prédicats ne peuvent renvoyer qu'à des collectifs, et n'auraient pas de validité dans le sens contraire. De la même manière, il semble que le prédicat «commettre un génocide» peut difficilement ne renvoyer qu'à une seule personne.

Mais testons ce point en prenant les choses à l'inverse: y aurait-il un sens à dire qu'une personne seule «commet un génocide»?

$\mathrm{Si}$ on réfère exclusivement à la définition du génocide telle qu'elle apparaît dans la Convention des Nations Unies de 1948, autrement désignée comme «la Convention sur le génocide», il semble qu'effectivement cela soit envisageable. Le génocide y est défini comme «un crime imprescriptible commis dans l'intention de détruire en tout ou partie un groupe national, ethnique, racial ou religieux». L'élément ambigü de cette définition est, pour la question qui nous intéresse ici, la précision «en tout ou partie». En effet, dès lors qu'on reconnaît qu'on ne peut faire de distinction pertinente, entre "tous moins un», puis entre «tous moins deux», nous pouvons retrancher ainsi des unités jusqu'à ce qu'il n'y en ait plus qu'une. Force est alors d'admettre que la définition du génocide s'applique à toutes les tailles effectives de parties, et ne dépend pas structurellement du nombre des victimes. Nous pouvons légitimement penser, selon la définition donnée ici, un génocide qui ne toucherait qu'une seule personne.

Dès lors, l'élément le plus important de la définition serait non pas le caractère étendu du crime comme nous aurions pu le penser spontanément, mais bien "l'intention de détruire [...] un groupe national, ethnique, racial ou religieux». Ce qui fait le génocide, et donc le génocidaire, serait proprement d'avoir commis une exaction visant l'extinction d'un groupe déterminé et dans lequel la victime n'a été choisie pour nulle autre raison qu'elle appartient à ce groupe. 
Mais alors, si le nombre des victimes n'est pas pertinent et que ce qui compte est la nature de l'intention, qu'est-ce qui distingue, sur le plan individuel, l'acte de génocide du crime raciste?

Cette question est fondamentale pour établir la dimension collective du crime de génocide. Définissons rapidement le crime raciste comme un ou plusieurs homicides, motivés exclusivement par des signes d'appartenance de la victime à une race ou à une ethnie autre que celle à laquelle appartient le criminel. Ici, le mobile du crime est une haine envers un groupe donné, quelle qu'en soit la raison. Si la victime n'est choisie qu'en raison de son appartenance à une communauté, c'est en fait cette communauté qui est visée à travers un crime particulier, et non la personne. Il apparaît alors que ce crime révèle une volonté implicite de voir cette communauté disparaître. Quelle est alors la différence, au strict plan de la description des actes individuels, entre un acte de génocide et un crime raciste? Dans les deux cas, un individu a tué (et nous avons vu que le nombre de victime n'était pas l'élément central pour conclure au crime de génocide), dans les deux cas, l'intention qui a mené à l'action était la destruction d'un groupe déterminé. Il semble effectivement y avoir identité, si nous canalisons notre attention sur les actes propres du criminel, entre les deux crimes. D'ailleurs, le droit lui-même s'y perd: si nous avons vu que la définition juridique du génocide pouvait admettre les exactions commises contre une seule victime, une juriste comme Anne-Marie La Rosa (2003 : 393) nous fait remarquer que le crime raciste, parce qu'il est une atteinte «en relation avec l'intention de détruire le groupe lui-même», semble pouvoir être accepté juridiquement au titre de génocide.

Il semble qu'il y ait quand même une différence de taille entre le génocide et le crime raciste, différence de l'ordre de celle qu'il y a entre l'intention et la commission. Car si nous pouvons dire que le criminel raciste manifeste par son geste une volonté plus ou moins consciente de voir un groupe disparaître, à fortiori s'il revendique clairement son crime comme un incitatif, il sait que ce n'est pas effectivement le cas. Nous allons voir que le génocidaire, lui, est suffisamment conscient (c'est la condition) que d'autres, en même temps que lui, se livrent aux mêmes actes.

Comme le fait remarquer Anne-Marie La Rosa (2003), il n'est pas suffisant que les actes soient concomitants au génocide pour qu'on puisse les classer comme actes de génocide, il faut encore que la conscience de participer à un génocide soit avérée. En d'autres termes, si un 
individu, M. X, commet un crime raciste, et s'aperçoit ensuite que le groupe auquel appartient sa victime vient justement, le même jour et dans la même zone, d'être victime d'une attaque généralisée qu'on peut qualifier de génocide, nous ne pourrons pas conclure pour autant que $\mathrm{M}$. X est coupable de participation à un génocide, mais bien qu'il est coupable de crime raciste.

L'élément intentionnel du génocide ne peut donc se limiter au fait de viser la destruction physique d'un groupe, il doit également contenir, au moins de façon larvée, la conscience de participer effectivement à une telle entreprise.

Si nous ne nous en tenons qu'à la seule description des actes, les crimes du génocidaire et du criminel raciste peuvent recevoir la même description, mais le contenu de l'intention et la connaissance des conséquences liées à son acte propre ne sont pas les mêmes.

Deux raisons nous permettent donc de conclure que le crime de génocide est bien un crime nécessairement collectif: l'élément intentionnel du crime ne contient pas uniquement la volonté de détruire un groupe pour des raisons racistes, il comprend également la conscience que d'autres crimes semblables sont en cours. Ce point esquisse déjà une forme de connexion entre les différents crimes. De plus, comme conséquence de ceci, le crime de génocide possède nécessairement une certaine extension matérielle, qui n'est pas due au nombre des victimes comme nous l'avons établi, mais à cette connaissance que d'autres personnes sont effectivement en train de commettre les mêmes actes.

\section{Quel type de collectif est-il contenu dans le crime de génocide?}

La nature du collectif en jeu dans le crime de génocide nous est nécessaire pour comprendre quel type de liaison structurelle existe entre les génocidaires.

Nous l'avons esquissé, un crime quelconque connaît toujours deux dimensions: les actes purs ou l'actus reus et la conscience psychologique du crime ou mens rea. Il nous faut donc examiner successivement ces deux aspects.

Quel est le caractère collectif de l'actus reus?

Nous l'avons vu, le génocide doit logiquement avoir une certaine extension. Là encore, mais dans une optique inverse, nous nous heurtons au 
problème ontologique de l'évaluation du nombre: à partir de combien de crimes particuliers pouvons-nous précisément parler de génocide? Et dès lors, est-ce que le crime de génocide est un crime collectif distributif, c'est-à-dire est-il constitué de l'addition des exactions particulières?

Que ce soit en ex-Yougoslavie ou au Rwanda, il y a d'abord eu enquête pour savoir s'il y avait eu génocide ou non et si le terme était approprié à ce qui s'était passé. «Dans leur examen de fond des affaires, les Chambres ont estimé nécessaire, dans un premier temps, de vérifier si un génocide avait effectivement eu lieu en avril 1994 au Rwanda, abstraction faite de toute responsabilité pénale individuelle» (La Rosa, 2003 : 411). Dès lors, il semble que ce qui permet de conclure au génocide n'est pas l'addition effective de crimes, puisque d'un point de vue logique, on devrait déjà avoir jugé les prévenus pour ensuite additionner le nombre de coupables et conclure au-delà d'un certain seuil au crime collectif. Mais ceci n'est pas indispensable. L'état de «santé globale» d'un groupe ethnique avant et après des événements sociaux semble être un premier indicateur. Des présomptions lourdes et concordantes de la commission d'un nombre éloquent d'exactions permettent de conclure au génocide en dehors de l'examen de tout cas particulier.

Au Rwanda, on a longtemps parlé «d'actes de génocides» avant de reconnaître «un génocide», on a mis du temps (constatons-le sans en commenter ici les raisons) à reconnaitre une valeur d'ensemble. C'est à ce niveau-ci que le caractère étendu des crimes reprend de l'importance: le fait de conclure à un génocide ou à des actes de génocide dépend en bonne part des mesures prises à grande échelle et du nombre important de victimes. Le nombre, qui ne pouvait logiquement intervenir comme élément déterminant dans l'énonciation en raison de la possibilité de régression à l'infini qu'il permet, intervient pourtant de manière centrale dans la définition pragmatique des actes. Un génocide est un crime à grande échelle, et il convient pour certains auteurs, dont Anne-Marie La Rosa, de réserver le terme aux cas les plus graves afin de ne pas galvauder un crime qui peut paraître à divers égards comme le pire des crimes possibles.

C'est ensuite, dans un second temps, une fois qu'a été reconnu un crime global de génocide, qu'on pourra subsumer un nombre, non encore défini, d'actes de génocides sous le chef de génocide. Il faut donc bien distinguer «le génocide» proprement dit, crime collectif, des «actes de génocides», crimes particuliers. 
Pour comprendre cette distinction, nous pouvons en revenir à la position de Peter French, exposée précédemment, pour faire une analogie entre le prédicat «élire un président» et «commettre un génocide». En effet, ce n'est pas chaque personne qui élit un président, chaque personne vote et l'addition des votes décide de l'élection de tel ou tel candidat. À proprement parler, c'est bien le groupe qui élit. De la même manière ici, ce n'est pas chaque personne qui commet un génocide, chaque personne tue, viole, commet un certain nombre d'exactions, dont l'addition constitue un génocide. Mais là aussi c'est bien un groupe qui commet un génocide.

Dès lors, il semble que déjà au plan de la compréhension de l'actus reus, le génocide ne puisse se réduire à une collection d'actes particuliers. Un génocide est toujours plus que $\mathrm{x}$ meurtres, $\mathrm{x}$ viols, etc. Certes, dans les faits, c'est bien le caractère répétitif des crimes qui a permis de décider qu'il y avait génocide, mais cette décision n'est pas neutre. À partir du moment où on reconnaît le génocide, on admet un crime global indépendant des acteurs qui y ont participé et même des actes précis qui ont été commis.

Une fois ce caractère englobant reconnu, les actes particuliers des différents génocidaires vont venir se placer sous cet élément surplombant, qui devient dès lors une entité distincte des crimes particuliers.

Par conséquent, le génocide nous place face à un crime globalisant, constitué d'actes de génocides, mais dont le caractère collectif propre est plus que l'addition des actes particuliers.

\section{Quel est, maintenant, le caractère collectif de la mens rea?}

Nous l'avons vu, pour accuser un individu de génocide, il faut faire la preuve d'une dimension intentionnelle collective du crime. Mais comment considérer le caractère intentionnel d'un groupe? Et comment en faire la preuve?

Parler d'intentionnalité collective peut d'abord sembler antinomique dans la mesure où une intention, à proprement parler, réfère à des capacités mentales de représentation et de décision qui ne peuvent renvoyer qu'à l'individu et non à un groupe. C'est pourquoi certains auteurs, comme Max Weber (1978) ou H. D. Lewis (1948) ne reconnaissent pas d'intention aux groupes. Ceci s'explique en partie par le fait qu'ils n'acceptent qu'une seule forme d'intentionnalité, et en viennent donc logiquement à constater que la définition de l'intention valable pour un agent autonome ne peut convenir à des agents collectifs. 
Cependant, nous allons voir qu'il est possible d'admettre d'autres formes d'intention

Tout d'abord, nous pouvons tout à fait penser une intention collective comme la somme des intentions concordantes de tous les membres du groupe. Par exemple, imaginons une association de malfaiteurs se mettant d'accord pour commettre un crime. Tous visent le même but et vont agir de concert pour parvenir à leur fin, se rendant ainsi les coauteurs du crime. L'intention peut donc être vue comme l'intention collective de l'association.

On pourrait d'abord objecter que ce qui est valable pour une association de malfaiteurs ne peut l'être pour des génocidaires, car ces derniers ne se rencontrent pas pour concevoir par consensus un projet génocide.

Mais nous pouvons répondre qu'il n'y a là qu'une impossibilité pratique et en rien une impossibilité logique. À partir du moment où l'on accepte que deux individus puissent avoir la même intention et s'associer afin de réaliser cette intention, nous pouvons considérer qu'est constitué un groupe ayant une intention unique, celle-ci étant à penser comme l'addition des intentions particulières. À partir de là, le nombre de personnes qui appartiennent au groupe est contingent, et nous pouvons penser un groupe de millions de personnes ayant toutes absolument la même intention. Certes, dans les faits, il est pour ainsi dire impossible de voir apparaitre ce genre de chose, mais ce n'est pas logiquement incohérent.

Nous avons ainsi mis au jour une première forme d'intention collective qui serait à penser comme la concordance de plusieurs intentions identiques.

Est-ce le type d'intention collective qui serait en jeu dans le crime de génocide? Il apparaît rapidement que ce n'est pas le cas, mais ce prédicat n'est pas écarté pour des raisons pratiques. Nous avons vu dans notre partie précédente qu'il n'était pas nécessaire de compter les crimes particuliers pour décider s'il y avait ou non eu génocide, de la même façon et pour les mêmes raisons, il n'est pas non plus nécessaire d'additionner les intentions concordantes pour juger si les crimes commis l'ont bien été avec l'intention de détruire tout ou une partie d'un groupe donné.

Un autre élément corrobore cette idée: que l'actus reus et la mens rea du crime doivent être commensurables. Il semble logiquement incohérent d'avoir une mens rea de nature collective distributive si l'actus reus 
ne l'est pas. Si le crime de génocide possède une dimension globalisante sur le plan de l'actus reus, on doit retrouver cette dimension globalisante aussi dans la mens rea. L'intentionnalité collective propre au crime de génocide n'est donc pas à penser comme l'addition des intentions concertées de ses membres et est dès lors à chercher dans une autre direction.

La seconde façon d'envisager l'émergence d'une intention collective est de la considérer structurée par quelques-uns et proposée ensuite au groupe qui l'accepte de manière libre ou autoritaire.

Dans le premier cas, le groupe se forme comme un agglomérat autour d'un projet commun. Nous pouvons alors considérer que l'adhésion à un groupe comporte un accord implicite aux projets et aux actes de ce groupe. Dans le cas du génocide, le groupe se constitue par tous ceux qui prennent l'initiative de participer à des actes de génocide.

Dans le second cas, l'intention organisée par ceux qui sont dépositaires de l'autorité est alors proprement une politique. Le groupe est alors implicitement constitué: il s'agit de ceux à qui est imposée cette autorité et qui peuvent éventuellement risquer leur propre vie en refusant de participer au génocide.

C'est bien à ce type d'intentionnalité collective qu'il faut rapporter l'intentionnalité en jeu dans le crime de génocide. Le génocide qui a eu lieu au Rwanda en 1994 se rapprocherait plus du premier type, le génocide juif de la dernière Guerre mondiale se rapprocherait plus du second. Mais ces distinctions nous permettent surtout de révéler deux intentions qui se superposent plus ou moins suivant les circonstances: l'intention du groupe, qu'on peut désigner comme l'intention collective, et l'intention individuelle, qu'on va juger à l'aune de la première.

Cependant, nous ne sommes pas encore pour autant au bout de nos peines, car il faut maintenant déterminer quelle est l'intention qui vaudra comme intention collective. Or la volonté de détruire un groupe donné est rarement aussi clairement affirmée et aussi organisée que pendant la Seconde Guerre mondiale. Dans des cas comme l'ex-Yougoslavie ou le Rwanda, est-ce qu'on peut identifier une politique, un représentant ou même un projet? Un appel au génocide comme on en a, par exemple, entendu sur la radio des mille collines au Rwanda peut-il être compris comme la cristallisation d'une intention collective?

Les difficultés inextricables dans lesquelles nous plongent ces questions expliquent que, parfois, on doit inférer une intention collective d'un comportement général. Si bien que ce qui est considéré comme 
l'intention du groupe peut ne renvoyer effectivement à aucune intention individuelle et, c'est presque paradoxal, ne renvoyer finalement à aucune intention formulée. Comment alors, parler encore d'intention collective?

Nous pouvons nous appuyer ici sur le caractère particulier du comportement collectif. L'action collective, comme toute action, renvoie à une forme d'intention dans laquelle on peut lire des croyances et des désirs. Nous pouvons alors inférer, d'une baisse soudaine et non naturelle du nombre de ressortissants d'un groupe donné, que les crimes systématiquement dirigés contre eux révèlent une volonté globale de les voir disparaitre. Dans ce cas, on remonte d'un comportement collectif aux croyances et désirs qui y correspondent. Nous pouvons donc tout à fait penser une intention qui ne renvoie qu'au groupe et non à personne ni à aucun discours particulier.

Ainsi, le crime de génocide nous demande de penser une forme d'intentionnalité collective qui serait celle du groupe comme entité abstraite, renvoyant à un second examen la question de savoir quelle est l'intention effective de chacun de ses membres.

De cette manière, nous avons mis au jour que le crime de génocide possédait, tant sur le plan de l'actus reus que de la mens rea, une dimension globalisante, distincte des actes et des intentions particulières. C'est en vertu de cette dimension globalisante qu'il y a en chaque acte de génocide quelque chose qui outrepasse les simples conditions de sa commission et qui le distingue du crime raciste.

\section{De la structure collective du crime de génocide à l'établissement des liens structurels entre les génocidaires}

Nous avions vu dans la première partie que l'intention du génocidaire devait contenir, au moins de façon rudimentaire, la connaissance que d'autres crimes avaient cours ailleurs et la conscience minimale de participer à une entreprise systématique. Une forme de liaison entre les crimes et entre les génocidaires avait commencé à s'esquisser. Dans la seconde partie, nous avons établi que le crime de génocide est plus qu'un crime collectif distributif et possède une dimension englobante, tant sur le plan de l'actus reus que de la mens rea. Il nous faut donc à présent remettre ceci en perspective et établir la structure de la collectivité afin de comprendre comment les génocidaires peuvent être liés entre eux. 
Afin de modéliser les structures possibles, il est nécessaire de considérer ici le génocidaire lambda comme en pleine connaissance des événements et de la portée de ses actes, et comme adhérant totalement au projet général de destruction d'un groupe déterminé. Nous réserverons l'évaluation de sa responsabilité pour la dernière partie.

Pour le moment, comprendre quelle est la structure collective contenue dans le génocide nous demande de nous interroger sur le type d'organisation que suppose un génocide.

La structure du collectif en jeu dans le génocide est-elle dépendante de l'organisation matérielle du groupe?

Dans La dynamique des groupes restreints, Anzieu et Martin (1968: 75) ont distingué cinq formes de rassemblement: la bande, la foule, le groupement, le groupe primaire (ou restreint) et le groupe secondaire (ou organisation). Il apparaît rapidement que les syntagmes «la bande», «le groupement» et «le groupe primaire», en raison du nombre très limité de personnes qu'ils supposent, ne sont pas adéquats pour rendre compte du type d'organisation pouvant perpétrer un génocide. Seuls un groupe ou une foule de type «groupement secondaire» semblent susceptibles de commettre ce type de crime. La différence entre ces deux types de collectif est le degré de structuration fonctionnelle qui existe dans les groupes secondaires, alors qu'une foule est un agglomérat plus ou moins aléatoire d'individus autonomes.

Un de ces deux types de collectif est-il propre au génocide à l'exclusion de l'autre?

Les exemples historiques nous montrent que nous pouvons grossièrement rapprocher le collectif du génocide Rwandais de la foule et celui de la Seconde Guerre mondiale du groupe secondaire, car celui-ci revêt un degré d'organisation qu'on ne retrouve pas dans le premier. Ceci démontre que le génocide ne peut être perpétré par n'importe quel type d'organisation et dans le cas de celles qui sont susceptibles de s'y livrer, il n'y a aucun lien nécessaire entre des actes de génocide et un type d'organisation en particulier. Le collectif que représente l'ensemble des génocidaires n'est pas inhérent à un type de rassemblement plutôt qu'à un autre.

Dès lors, puisqu'on peut admettre qu'un génocide soit commis par une collectivité ayant aussi peu de cohésion interne qu'une foule, il ne 
semble pas, sur le plan de l'actus reus, qu'il y ait un degré minimal de structure du collectif des génocidaires. En effet, nous pouvons tout à fait imaginer des actes de génocides indépendants matériellement les uns des autres: chaque génocidaire se levant à l'appel d'un meneur raciste et se mettant à tuer ses voisins avec ce qui lui tombe sous la main. Dans un tel exemple, nous pouvons bien conclure au génocide puisque nous avons une quantité d'assassinats et qu'on peut dégager une intention collective permettant de laisser croire à chaque génocidaire que d'autres feront de même ailleurs. Mais nous ne constatons dans cette situation aucun lien matériel entre les actes.

Finalement, cette analyse nous permet de conclure que le degré d'organisation du groupe aura très certainement une sinistre influence sur le degré d'efficacité de l'entreprise, mais ne remet pas en cause la nature du génocide comme tel. Le type de structure matérielle exact, regroupant des individus dispersés et le groupe secondaire, est donc bien contingent.

Aussitôt, le lien qui peut exister entre les génocidaires n'est pas un lien matériel. Il peut même n'exister aucun lien matériel. Par conséquent, la relation entre les génocidaires n'est pas à penser par principe comme du type de relation qu'il y a entre des complices.

Ceci corrobore l'idée esquissée précédemment que, dans le cas du génocide, nous avons moins affaire à un rassemblement physique qu'à une «communauté d'intérêts». Ce qui crée vraiment le groupe est un élément psychologique : l'intention commune. Il semble que nous ayons là une forme de groupe originale, qui ne correspond en rien à une forme qu'on a l'habitude de distinguer. Pour Larry May (1987: 55), le groupe n'est pas à penser comme quelque chose de transindividuel, c'est-à-dire de surplombant et d'englobant, mais comme quelque chose de créé par les interactions entre individus. Ce qui fait la vraie particularité du crime de génocide est que l'interaction entre les individus est à examiner sur le plan moral. En effet, est-ce que le crime aurait été le même, sans rien dire du fait qu'il n'aurait peut-être pas eu lieu, si chaque génocidaire n'avait pas toutes les raisons de supposer que d'autres étaient en train de commettre les mêmes crimes?

Le collectif des génocidaires serait à penser minimalement comme un collectif ayant cette particularité de révéler une forme d'interaction morale sans que corresponde nécessairement une interaction matérielle. Il faut donc éviter l'erreur de considérer les deux niveaux comme étant liés de manière causale. Par conséquent, il n'est pas nécessaire de cher- 
cher une forme de collaboration matérielle censée anticiper ou prouver une forme de communauté d'intérêt, on peut penser cette communauté d'intérêt sans qu'il n'y ait aucune interaction matérielle effective. Et c'est en ce sens que le crime de génocide possède une structure collective originale.

Ainsi, on a montré que le lien entre les génocidaires n'est pas un lien de l'actus reus, mais exclusivement de la mens rea.

Il apparaît de ceci que c'est la communauté d'intérêt qui fait que l'acte d'un seul génocidaire peut cristalliser et contenir une marque de tous les autres actes de génocide, même s'ils n'ont matériellement aucun rapport entre eux. En effet, ce lien moral sur le plan de la mens rea fait qu'on peut voir les actes des uns et des autres comme en partie dus aux circonstances. S'il y a vraiment volonté de détruire un groupe ethnique et début de commission, on peut penser que chacun aurait détruit la totalité du groupe seul, s'il l'avait pu. Un génocidaire, à la différence du criminel raciste, ne commet pas un crime, il commet un premier crime. Et dans une perspective globale, puisqu'il suppose que d'autres font de même ailleurs, il a conscience qu'en réalité, son crime est un nième crime. C'est ce qui fait qu'un individu ayant participé aux actes est alors autant un génocidaire pour avoir tué une personne, 20 ou 30000. Finalement, les actes perpétrés par un génocidaire individuel sont en partie circonstanciels, chacun massacre dans son périmètre d'action et aurait massacré indifféremment dans un autre lieu. Dans ce contexte, sous-tendu par une forme de communauté d'intérêts, et parce que chacun a la connaissance que le crime commun est en train d'être perpétré ailleurs et par d'autres, chacun devient le bras armé de tous les autres, et réciproquement tous les autres peuvent êtres vus comme autant de bras armés du génocidaire lambda: chacun fait sa part d'un vaste projet, le degré d'organisation effectif étant secondaire.

Ce caractère collectif de la mens rea dégage donc bien une responsabilité morale collective caractéristique du crime de génocide.

Nous allons voir maintenant que cette structuration particulière du groupe sur le plan moral n'est pas sans incidence quant à la responsabilité individuelle des génocidaires. 


\section{Conséquences de ces liens structuraux entre génocidaires pour la responsabilité individuelle}

Il apparaît clairement que les actes de génocide particuliers ne peuvent pas de manière appropriée être traités juridiquement comme des crimes racistes, car même sur le plan de la description des actes individuels, ils ne sont jamais que cela. Les actes des autres membres du groupe ne sont pas à penser sous le schème de la juxtaposition, chacun agissant à côté des autres. Au contraire, les actes s'appellent et se répondent les uns les autres. Le lien moral qui structure la relation des génocidaires a montré que ce renvoi aux autres crimes est un élément constituant du crime particulier. Par conséquent, la responsabilité du génocidaire contiendrait à la fois une responsabilité individuelle pour ses actes propres et une seconde dimension, toujours individuelle, qui porterait le renvoi aux actes commis par les autres membres comme par procuration et distinguerait proprement l'acte de génocide du crime raciste.

La structure du crime de génocide fait que ces deux dimensions sont unies dans un acte unique. Et c'est bien parce que la dimension collective est un élément constituant de la description du crime individuel que nous pouvons concilier la dimension individuelle et la dimension collective du crime de génocide au sein du procès individuel. En ce sens, la responsabilité du génocidaire semble être une forme originale de responsabilité.

Refuser d'en référer aux circonstances des actes et d'intégrer la dimension collective du crime de génocide dans la responsabilité individuelle, n'est donc pas seulement perdre un élément de lecture, c'est également tronquer la responsabilité véritable du génocidaire d'un de ses éléments constituants.

$\mathrm{Au}$ vu de ce que nous avons établi, nous devrions donc conclure qu'un procès pour génocide devrait toujours prendre en compte le contexte, et si celui-ci apparaît comme facteur aggravant, ce ne peut être que justice. Cependant, nous en arrivons à cette conclusion parce que nous en sommes restés, afin de modéliser les structures, au cas particulier où le génocidaire est en pleine connaissance du contexte et adhère parfaitement à ce que nous avions désigné comme l'intention commune. Il ne s'agit encore que d'un modèle idéal, et le passage à plus de pragmatisme nous demande de prendre en compte de nouveaux éléments. Comment ce modèle évolue-t-il quand on en revient à des situations moins manichéennes, et bien sûr, plus réelles? 
La réflexion nous demande maintenant de disjoindre les intentions individuelles et collectives. Nous avions imaginé un génocidaire qui avait pleine connaissance du contexte, et qui adhérait pleinement au projet. Deux éléments étaient sous-entendus: la connaissance et la liberté (puisqu'on suppose que seule une forme de contrainte peut nous faire participer à un génocide auquel nous ne donnons pas notre assentiment). Observons donc successivement ces deux nouveaux éléments.

La liberté d'abord, car nous avons vu qu'un génocide pouvait se dérouler dans le cadre d'une politique et, à ce titre, user envers des génocidaires potentiels d'une forme de coercition. Refuser d'obéir aux ordres dans un tel contexte se révèle souvent périlleux pour sa propre vie. L'adhésion à un génocide peut, à ce titre, être pensée comme tout crime de guerre (la dimension nationale ou internationale n'est pas ici pertinente) commis sous la contrainte ou sous la pression d'une formation hiérarchisée. La responsabilité du génocidaire n'a donc pas dans ce cas d'élément original par rapport à la responsabilité de tout autre criminel de guerre, les difficultés seront les mêmes.

Attardons-nous maintenant aux collectifs dont le principe moteur n'est pas externe mais plutôt interne comme, par exemple, les foules. L'idée est parfois avancée que la foule (ou le groupe) a des dynamiques propres agissant sur les individus comme de puissants incitatifs qui les poussent à commettre des actes qu' ils n'auraient jamais commis de façon autonome. Ce raisonnement devrait nous porter à considérer ce facteur comme un élément de déresponsabilisation. Mais un incitatif est-il une contrainte? Il semble bien qu'on reste ici dans la perspective d'une intention collective proposée et non imposée. Dès lors qu'il participe, «l'intention collective de la foule» devient celle de l'individu, et il est secondaire de savoir pourquoi il y a adhéré.

$\mathrm{Au}$ contraire, dans le cas de la contrainte autoritaire, intention collective et intention individuelle peuvent rester disjointes. La difficulté, et elle est énorme, sera alors d'évaluer le degré d'adhésion du criminel à la politique commune.

Mais l'élément fondamental pour être coupable de génocide est sans doute d'avoir la connaissance de participer à un génocide. Par exemple, imaginons un individu qui participe à ce qu'il sait être un génocide, mais dont l'intention profonde n'est pas tant de détruire un groupe que de s'emparer des biens de son voisin. Cet individu sera bien coupable de génocide et non de meurtre. Pourtant il n'y a pas réelle intention de détruire un groupe ethnique, son intention propre devrait faire de son 
crime ni un acte de génocide ni même un crime raciste mais uniquement un crime ordinaire. Or s'il a conscience des événements et sait que son acte, quel que soit son mobile propre, fait le jeu d'un projet collectif de génocide, alors l'intention qui prévaut est l'intention collective de génocide. Du moment qu'on arrive à prouver que l'individu était en mesure de comprendre un projet, il n'a plus que deux choix: y adhérer ou le refuser, mais on ne peut accepter l'excuse d'une participation sous-tendue par des intentions dissidentes. La question n'est pas de savoir quelle est exactement l'intention de l'accusé, mais d'évaluer son degré d'adhésion à l'intention collective, et ce degré d'adhésion passe par le degré de connaissance et de compréhension de cette intention collective.

Finalement, l'intention collective apparaît concrètement dans le procès individuel comme une mesure permettant d'évaluer l'intention de l'accusé.

Par conséquent, pour être pleinement responsable, le génocidaire doit non seulement être suffisamment informé, mais aussi être suffisamment mature pour comprendre que les conséquences de ses actes vont pouvoir s'inscrire dans un crime plus vaste. Un manque dans l'une ou l'autre de ces dimensions appelle une diminution de la responsabilité. Ceci suppose deux choses. D'une part, qu'il faut, pour être convaincu de génocide, un degré de maturité suffisant. Cette remarque peut sembler superflue dans la mesure où ceci est valable pour tout crime. Mais justement, cela doit être vrai pour tout crime. Cette observation vise à écarter la proposition que, compte tenu de la gravité du crime, même les enfants seraient conscients de leurs actes, et qu'il est opportun dans un cas comme celui-là d'abaisser l'âge de la responsabilité légale. Or la gravité du crime ne peut être a priori un facteur responsabilisant, ce serait prendre les choses à l'envers: inférer la subjectivité d'une faute et non évaluer la faute à l'aune de la subjectivité. La responsabilité de l'accusé doit bien être évaluée à partir de ses capacités, sans que l'horreur du crime n'intervienne comme facteur responsabilisant.

Le second élément lié à la connaissance est que ceux qui ne savaient pas et ne pouvaient pas savoir pour une raison ou pour une autre ce qui était en train de se passer ne peuvent être accusés de génocide mais, suivant leur mobile, de crime simple ou de crime raciste. Et c'est finalement le point déterminant: tout l'enjeu du procès va être d'évaluer le degré de concomitance entre l'intention collective et l'intention individuelle. 
La grande difficulté sera alors de proposer une échelle de proportionnalité entre un degré de connaissance imparfaite et un degré de responsabilité.

\section{Pour conclure}

La nature collective particulière du crime de génocide nous a permis de montrer que les génocidaires entretenaient entre eux une forme de lien relatif à la dimension non distributive du crime et fondé sur la notion d'intention collective. Le renvoi aux actes commis par d'autres nous est apparu comme un élément constituant de chaque acte particulier. La conséquence de cette structure était que tous les actes de génocide s'enchâssaient les uns dans les autres, non pas nécessairement de manière causale, mais d'une façon originale telle que chaque acte contient tous les autres de manière intrinsèque. Cette particularité du crime de génocide fait que, bien qu'étant de nature collective, il puisse être rendu de manière appropriée dans un jugement individuel. Par conséquent, il ne faut pas craindre, lors du procès, de mettre l'acte particulier en perspective avec le contexte, et faire le contraire revient à le priver de sa dimension originale et à perdre ce qui, dans le génocide, excède le crime raciste. Il est donc tout à fait attendu que cette perspective entraîne un alourdissement de la responsabilité sur le plan individuel.

Cependant, nous l'avons vu, nous pouvons soutenir cette conclusion lorsque nous nous mettons dans le cas particulier d'une intention individuelle concordant parfaitement avec l'intention collective. Or, d'un point de vue concret, nous avons vu que la responsabilité du génocidaire, comme toute responsabilité, pouvait laisser place à des éléments déresponsabilisants. De plus, d'un point de vue pratique, il est très difficile d'établir quelle est précisément l'intention de l'individu et d'en faire la preuve. C'est ce qui explique que les individus inculpés et condamnés pour génocide sont presque toujours des gens dont l'intention est plus facilement reconnaissable en raison de leur position de pouvoir: par exemple, parmi les personnes condamnées à ce jour au Tribunal pénal international pour le Rwanda (TPIR), se trouvent un premier ministre, un préfet, un bourgmestre, un animateur radio, etc. Il convient donc de garder à l'esprit un principe de prudence lorsque nous tenterons de passer du modèle théorique à l'application pratique. Du 
moins, avons-nous un modèle étalon qui nous prouve que la réconciliation du caractère collectif et du caractère individuel au sein du jugement est possible et que l'entorse au principe de l'individualité de la peine n'est en rien une nécessité?

\section{Références}

Anzieu, D. \& Martin, J.-Y. (1968). La dynamique des groupes restreints. France: Presses universitaires de France.

French, P. (1998). Individual and Collective Responsibility. Rochester, VT: Schenkman.

La Rosa, A.-M. (2003). Juridictions pénales internationales, la procédure et la preuve. France: Presses Universitaires de France.

Lewis, H. D. (1948). Collective Responsibility. Philosophy, 24, (3), 1.

May, L. (1987). The Morality of Groups. Notre-Dame:University of Notre-Dame Press.

Weber, M. (1978). Economy and Society. California: University of California Press. 